\title{
The singlet model of the anisotropic magnetization of the III-VI diluted magnetic semiconductor, $\operatorname{In}_{1-x} \mathbf{M n}_{x} \mathrm{~S}$
}

\author{
G. Franzese, Ashlee Byrd, J. L. Tracy, J. Garner, ${ }^{\text {a) }}$ and T. M. Pekarek \\ Department of Chemistry and Physics, University of North Florida, Jacksonville, Florida 32224 \\ I. Miotkowski and A. K. Ramdas \\ Department of Physics, Purdue University, West Lafayette, Indiana 47907-2036
}

(Presented on 11 November 2004; published online 5 May 2005)

\begin{abstract}
Results for the anisotropic magnetization of the III-VI diluted magnetic semiconductor (DMS), $\mathrm{In}_{1-x} \mathrm{Mn}_{x} \mathrm{~S}$, are presented. The compound has a markedly different crystal structure from previously investigated III-VI crystals. The Hamiltonian includes crystal potential, Zeeman, spin-orbit, and spin-spin terms. The singlet model used assumes that the substitutional $\mathrm{Mn}$ are noninteracting which is appropriate when $x$ is small (here 2\%). Magnetization versus temperature results are found for several magnetic fields $\mathbf{B}$. The experimental magnetization is compared to our singlet model results with excellent agreement except at low temperatures $(\leqslant 20 \mathrm{~K})$ where some evidence of possible spin-glass behavior is evident. (C) 2005 American Institute of Physics. [DOI: 10.1063/1.1847451]
\end{abstract}

\section{INTRODUCTION}

A calculation and measurement of the magnetization of the III-VI diluted magnetic semiconductor (DMS), $\mathrm{Ga}_{1-x} \mathrm{Mn}_{x} \mathrm{~S}$, have recently been carried out with excellent agreement between theory and experiment. ${ }^{1}$ This class of DMS crystals is prepared by adding trace amounts of a transition-metal atom to the III-VI host. It is assumed that the transition-metal atoms enter the crystal by randomly substituting for some of the group-III atoms and give rise to the magnetization of the sample. A number of promising electrooptical applications have been proposed for III-VI materials due to their nonlinear optical properties. ${ }^{2,3}$ The III-VI DMS materials represent a relatively unexplored class of materials especially from a theoretical standpoint.

This paper presents model and measurement results for the magnetization of $\operatorname{In}_{1-x} \mathrm{Mn}_{x} \mathrm{~S}$, a new member of the III-VI DMS class that has a markedly different crystal structure from previously studied systems. ${ }^{4}$ The magnetization was calculated for applied fields with several orientations relative to the underlying lattice including parallel and perpendicular directions relative to the $c$ axis. The calculated magnetization exhibited significant anisotropy and for these two extreme field directions the results were generally found to bracket the measured magnetization. By suitable angular averaging, which is justified based on the crystal structure, the agreement between experiment and model magnetization gives excellent agreement for temperatures above about $20 \mathrm{~K}$.

\section{MODEL HAMILTONIAN}

To determine the magnetization we begin by finding the $d$-electron energy levels of the transition-metal atom (Mn) inside the III-VI (InS) crystal in the presence of magnetic field, B. Presumably the substitutional Mn atoms are bonded to the four nearest-neighbors atoms via covalent bonds. ${ }^{5}$ The incomplete $3 d$ valence shell of the Mn gives rise to the mag-

${ }^{a)}$ Electronic mail: jgarner@unf.edu netic moment of the sample. The energy levels of the $d$ electrons of the $\mathrm{Mn}$ atom are perturbed by the crystal field. In this work only nearest-neighbor interactions are considered and the point-ion approximation is adopted. The $d$-electron energy levels are then determined by the crystal symmetry, distance between ions, bond angles, and the values chosen for the formal oxidation states of the ions.

The orthorhombic crystal structure of InS is shown in Ref. 6. The manganese ion resides at the center of an elongated tetrahedron with three $\mathrm{Mn}-\mathrm{S}$ bonds and one $\mathrm{Mn}-\mathrm{In}$ bond. The angle between the Mn-In bond and each of the $\mathrm{Mn}-\mathrm{S}$ bonds was taken to be tetrahedral $\left(\theta=109.5^{\circ}\right)$. Throughout half the crystal the Mn-In bonds have one orientation (say, $0^{\circ}$ ) while over the other half the Mn-In bonds make an angle of $70.53^{\circ}$ with respect to the first set of Mn-In bonds. The substitutional $\mathrm{Mn}^{+3}$ ion has a $3 d^{4}$ outer electron configuration and a ${ }^{5} D$ ground-state term according to Hund's rules.

Within the singlet model of noninteracting Mn ions the Hamiltonian for an isolated transition-metal ion in the semiconducting host has the well-known form, ${ }^{7}$

$$
H=H_{\text {free-ion }}+H_{\text {crystal }}+H_{\text {spin-orbit }}+H_{\text {spin-spin }}+H_{\text {Zeeman }} \text {, }
$$

where $H_{\text {free-ion }}$ is the Hamiltonian of the free $\mathrm{Mn}^{+3}$ ion. Standard expressions ${ }^{1,7}$ are used to represent the spin-orbit, $H_{\text {spin-orbit }}(\lambda)$, the spin-spin, $H_{\text {spin-spin }}(\rho)$, and the Zeeman Hamiltonian, $H_{\text {Zeeman. }}$ In the $\operatorname{In}_{1-x} \mathrm{Mn}_{x} \mathrm{~S}$ results presented below, the spin-spin coupling constant used was $\rho=0.18 \mathrm{~cm}^{-1}$ (Ref. 7) and the spin-orbit coupling constant used was $\lambda$ $=7.8 \mathrm{~cm}^{-1}$ (Ref. 7). Note, however, this $\lambda$ has been adjusted to account for the fact that $\mathrm{Mn}$ is not a free ion but exists inside a host.

The crystal-field term of the Hamiltonian in Eq. (1) is found to have the operator equivalent representation, 


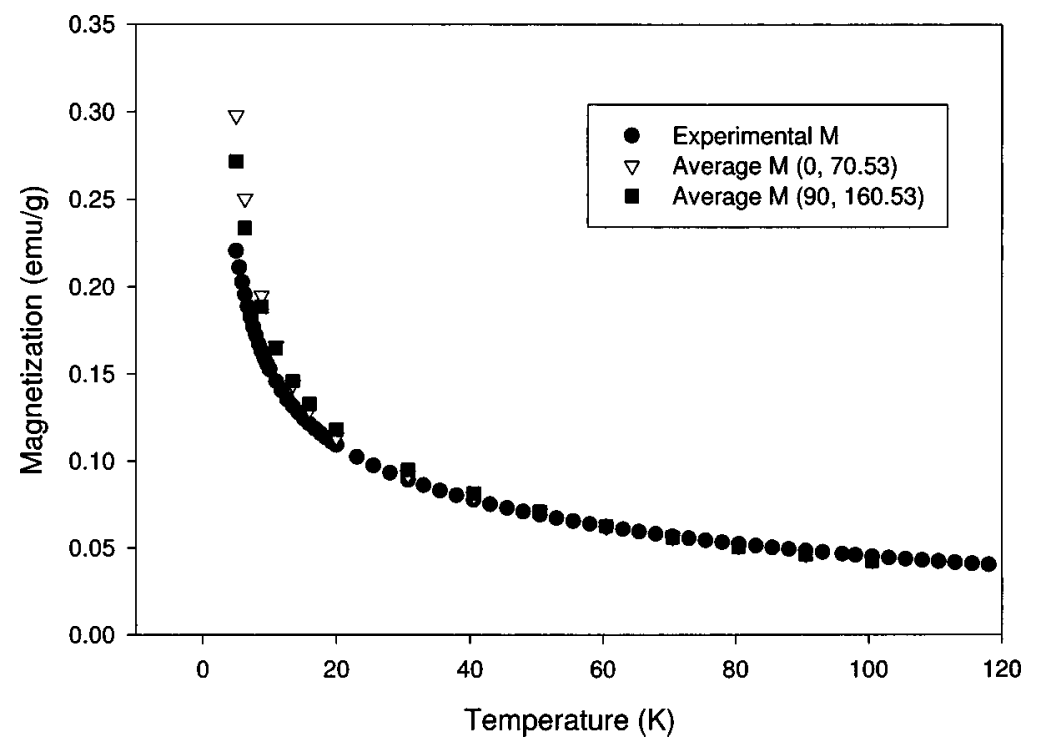

FIG. 1. Magnetization vs temperature of $\operatorname{In}_{1-x} \mathrm{Mn}_{x} \mathrm{~S}$ with nominal $x=2 \%$ in a magnetic field of $1 \mathrm{~T}$. The filled dots represent the measured magnetization, while the other two curves are angular averaged magnetization found from using the singlet model. From 120 to $400 \mathrm{~K}$ (not shown) the experiment and theory were indistinguishable.

$$
\begin{aligned}
H_{\text {crystal }}= & b\left[3 L_{z}^{2}-L(L+1)\right]+a\left\{35 L_{z}^{4}\right. \\
& +[25-30 L(L+1)] L_{z}^{2}+3 L^{2}(L+1)^{2} \\
& -6 L(L+1)\}-d\left\{L_{z}, L_{+}^{3}+L_{-}^{3}\right\} .
\end{aligned}
$$

Here $L_{ \pm} \equiv L_{x} \pm i L_{y}$ and $L_{x}, L_{y}$, and $L_{z}$ are the components of the total electronic orbital angular momentum operator along the Cartesian axes, $x, y$, and $z$ and $L(S)$ are the total orbital (spin) quantum numbers $(L=S=2$, for the ground term). Expressions for the coefficients, $a, b$, and $d$ are given elsewhere. ${ }^{1}$ A matrix representation of the Hamiltonian was obtained using the "uncoupled" angular momentum basis, $\left|L S M_{L} M_{S}\right\rangle$, with $L=S=2$ and both $M_{L}$ and $M_{S}=0, \pm 1, \pm 2$.

\section{COMPARISON TO EXPERIMENT}

A bulk single-crystalline $\operatorname{In}_{1-x} \mathrm{Mn}_{x} \mathrm{~S}$ sample with a nominal concentration $x=2 \%$ was taken from a boule. The sample was grown by the vertical Bridgman method and had a mass of $57.4 \mathrm{mg}$. Magnetization measurements were made between 1.8 and $400 \mathrm{~K}$ in fields up to $7 \mathrm{~T}$ using a Quantum Design MPMS XL7-superconducting quantum interference device (SQUID) magnetometer. The diamagnetic susceptibility of a pure InS crystal was measured to be -3 $\times 10^{-7} \mathrm{emu} / \mathrm{gG}$. This contribution to the magnetization has been substracted from the data.

The model magnetization is found using,

$$
M(T, B)=-\frac{n(x)}{Z} \sum_{i=1}^{N} e^{-\beta E} i \frac{\partial E_{i}}{\partial B} .
$$

In Eq. (3), $\beta=1 / k_{B} T$ (with $k_{B}$ the Boltzmann constant), $Z$ is the partition function, $N$ is the number of energy levels $(N$ $=25$ for Mn with $3 d^{4}$ ), $E_{i}$ is the electron energy level (eigenvalue of $H$ ), and $n(x)$ gives the number of Mn ions per unit mass of the sample for concentration $x$. Specifically,

$$
n(x)=x N_{A} /\left[(1-x) M_{\mathrm{In}}+x M_{\mathrm{Mn}}+M_{S}\right]
$$

where $M$ is the atomic mass of each constituent and $N_{A}$ is Avogadro's number.

From the reported crystal structure ${ }^{6}$ it is clear that half the In-Mn bonds are aligned along one direction (say, $0^{\circ}$ ) while the other half makes an angle of $70.53^{\circ}$ with the first

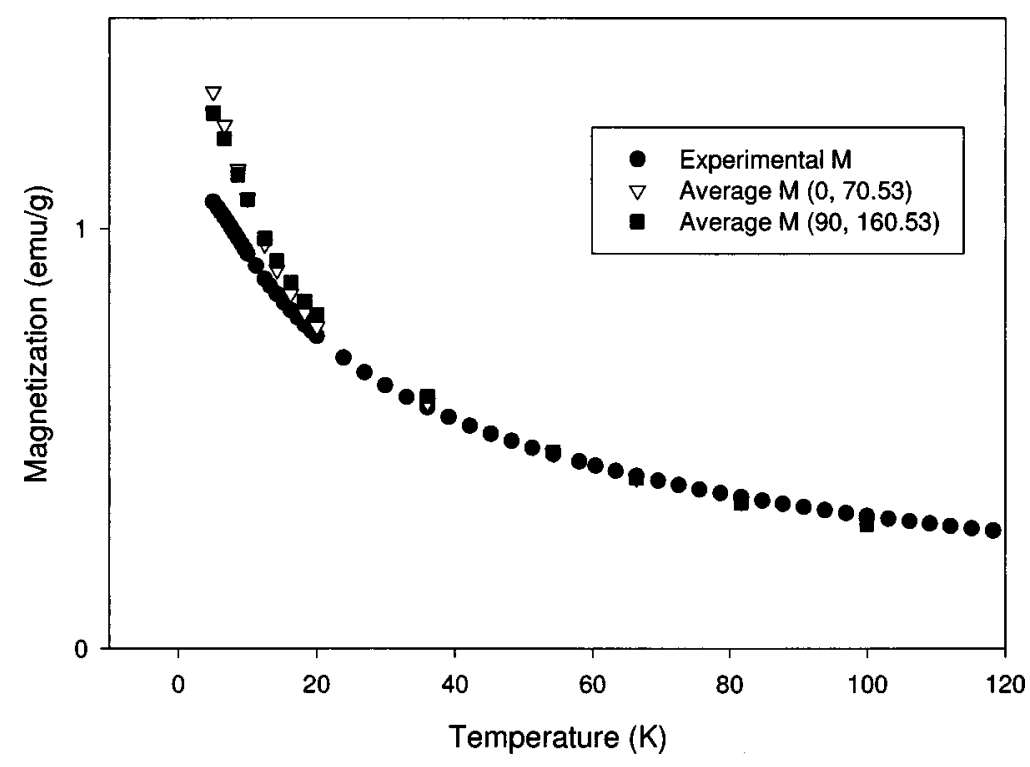

FIG. 2. Same as in Fig. 1, except the magnetic field is $7 \mathrm{~T}$. The experiment and theory agree from 120 to 400 $\mathrm{K}$ (not shown). 


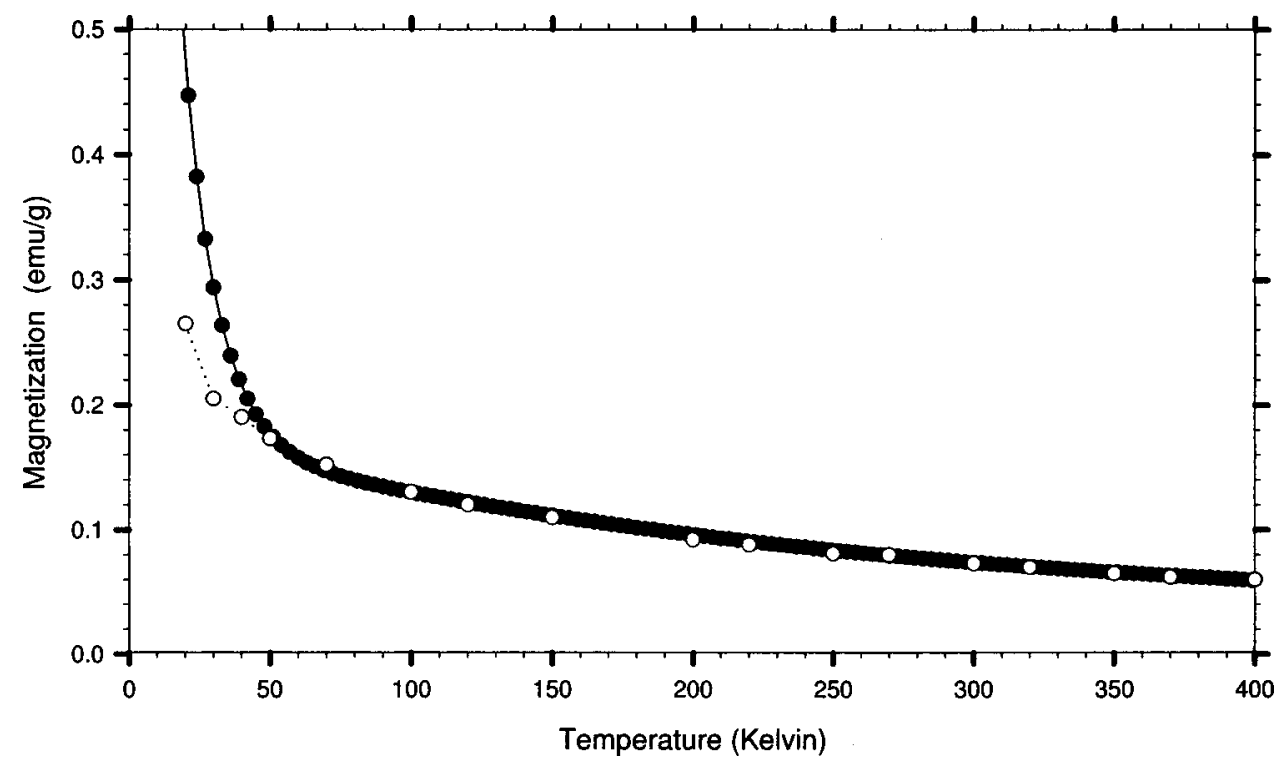

FIG. 3. Magnetization vs temperature of $\mathrm{Ga}_{1-x} \mathrm{Mn}_{x} \mathrm{~S}$ with $x=6.6 \%$ in a magnetic field of $1 \mathrm{~T}$. The open circles represent the experimental data and the filled circles are the singlet model results. The singlet model breaks down at about $50 \mathrm{~K}$

set. Another equally probable scenario has half the In-Mn bonds at $90^{\circ}$ and the other half at $\left(90^{\circ}+70.53^{\circ}=\right) 160.53^{\circ}$. The experiment did not attempt to align the crystal. In the calculations below, we have averaged the magnetization over angles assuming both possible scenarios i.e., $\left[M\left(0^{\circ}\right)\right.$ $\left.+M\left(70.53^{\circ}\right)\right] / 2$ and $\left[M\left(90^{\circ}\right)+M\left(160.53^{\circ}\right)\right] / 2$. The model results were found to be nearly the same in the two cases and are reported, along with the measured magnetization, in Figs. 1 and 2 . Figure 1 gives the magnetization versus temperature (up to $120 \mathrm{~K}$ ) for an applied field of $1 \mathrm{~T}$ and in Fig. 2 the field has a value of $7 \mathrm{~T}$. The agreement is excellent up to 400 $\mathrm{K}$, although the figures stop at $120 \mathrm{~K}$. The agreement begins to fail, however, at temperatures below about $20 \mathrm{~K}$.

The angular averaged magnetization calculations agree favorably with the data but begin to deteriorate as the temperature falls below about $20 \mathrm{~K}$. This behavior is reminiscent of similar behavior in the $\mathrm{Ga}_{1-x} \mathrm{Mn}_{x} \mathrm{~S}$ system ${ }^{1}$ shown in Fig. 3 where the breakdown in agreement between experiment and the singlet model was at about $50 \mathrm{~K}$. As in $\mathrm{Ga}_{1-x} \mathrm{Mn}_{x} \mathrm{~S}$, we believe the discrepancy may arise from the presence of a spin-glass transition. Similar arguments have been made for spin-glass behavior in certain II-VI DMS samples. ${ }^{8}$ In other samples of $\operatorname{In}_{1-x} \mathrm{Mn}_{x} \mathrm{~S}$ having a higher concentration of $\mathrm{Mn}$ $(>2 \%)$ a cusp appeared in the measured magnetization at about $5 \mathrm{~K}$. The prominent cusp found in $\mathrm{Ga}_{1-x} \mathrm{Mn}_{x} \mathrm{~S}$ was at a slightly higher temperature (about $11 \mathrm{~K}$ ) and there the singlet model began to deviate from the experiment at about four times the temperature of the cusp, i.e., near $50 \mathrm{~K}$. These numbers are consistent with the breakdown of the singlet model in $\operatorname{In}_{1-x} \mathrm{Mn}_{x} \mathrm{~S}$, also at about four times the temperature of the cusp, i.e., the breakdown occurs at $20 \mathrm{~K}$.

\section{CONCLUSION}

The first measurement and model of the magnetization of the III-VI DMS, $\operatorname{In}_{1-x} \mathrm{Mn}_{x} \mathrm{~S}$, has been performed. The crystal structure differs substantially from previously studied III-VI DMS structures. The magnetization was determined for several choices of the angle of the applied magnetic field. The magnetization was anisotropic. The magnetization provides a probe of the underlying electronic structure of the $d$ electrons of the transition-metal atom, Mn.

In all cases, the low-temperature theoretical singlet magnetization overestimates the experimental magnetization. An extension of the singlet model to incorporate Mn pairing via, e.g., antiferromagnetic superexchange interactions may help to extend the agreement with experiment to lower temperatures. The extension to incorporate Mn doublets is currently under way.

\section{ACKNOWLEDGMENTS}

This work was supported by UNF Research Grants, Research Corporation Award No. CC4845, Donors of the American Chemical Society Petroleum Research Fund PRF No. 40209-B5M, a Purdue University Academic Reimbursement Grant, and by the National Science Foundation (NSF) Grant Nos. DMR-03-05653, DMR-01-02699, and ECS-01-29853.

${ }^{1}$ C. Fuller, A. Douglas, J. Garner, T. M. Pekarek, I. Miotkowski, and A. K. Ramdas, Phys. Rev. B 65, 195211 (2002).

${ }^{2}$ S. Nusse, P. H. Bolivar, H. Kurz, F. Levy, A. Chevy, and O. Lang, Phys. Rev. B 55, 4620 (1997).

${ }^{3}$ S. Lee, S. Hahn, C. Chung, S. Yun, and W. Kim, Solid State Commun. 60, 453 (1986).

${ }^{4}$ T. M. Pekarek, C. Maymi, J. Garner, D. Hall, I. Miotkowski, and A. K. Ramdas, J. Appl. Phys. 91, 7496 (2002).

${ }^{5}$ S. Jandl, J. L. Brebner, and B. M. Powel, Phys. Rev. B 13, 686 (1976)., see also, M. Schluter and M. L. Cohen, ibid. 14, 424 (1976).

${ }^{6} \mathrm{See}$, for example, www.webelements.com for the InS structure.

${ }^{7}$ A. Abragam and B. B. Bleaney, Electron Paramagnetic Resonance of Transition Ions (Oxford University Press, New York, 1971).

${ }^{8}$ P. M. Shand, A. D. Christianson, T. M. Pekarek, L. S. Martinson, J. W. Schweitzer, I. Miotkowski, and B. C. Crooker, Phys. Rev. B 58, 12876 (1998). 